\title{
Orthogonal enzymatic reactions for rapid crosslinking and dynamic tuning of PEG-peptide hydrogels
}

\author{
Matthew R. Arkenberg and Chien-Chi Lin* \\ ${ }^{1}$ Department of Biomedical Engineering, Purdue School of Engineering \& Technology, Indiana \\ University-Purdue University Indianapolis, Indianapolis, IN 46202, USA
}

\footnotetext{
*To whom correspondence should be sent:

Chien-Chi Lin, PhD.

Associate Professor

Department of Biomedical Engineering

Purdue School of Engineering \& Technology

Indiana University-Purdue University Indianapolis

Indianapolis, IN 46202

Phone: (317) 274-0760

Email: lincc@iupui.edu
}

This is the author's manuscript of the article published in final edited form as:

Arkenberg, M. R., \& Lin, C. C. (2017). Orthogonal enzymatic reactions for rapid crosslinking and dynamic tuning of PEG-peptide hydrogels. Biomaterials science, 5(11), 2231. http://dx.doi.org/10.1039/C7BM00691H 


\begin{abstract}
Stiffening of the extracellular matrix is a hallmark in cancer progression, embryonic development, and wound healing. To mimic this dynamic process, our work explored orthogonal enzymatic reactions capable of modulating properties of poly(ethylene glycol) (PEG)-peptide hydrogels. A hepta-mutant bacterial transpeptidase Sortase $A\left(\mathrm{Srt}_{7 \mathrm{M}}\right)$ was used to ligate two PEG-peptide macromers (i.e., PEG-YLPRTG and $\mathrm{NH}_{2}$-GGGG-PEG) into a primary hydrogel network. The hydrogels were dynamically stiffened using mushroom tyrosinase (MT), which oxidized tyrosine residues into di-tyrosine and led to increased matrix stiffness. After confirming the expression and enhanced catalytic activity of $\mathrm{SrtA}_{7 \mathrm{M}}$, we investigated the cytocompatibility of the enzymatic reaction with a mouse insulinoma cell line, MIN6. In addition, we altered peptide substrate concentrations and evaluated their influence on primary hydrogel network properties and MT-triggered stiffening. Using a pancreatic cancer cell line, COLO-357, the effect of MTtriggered stiffening on spheroid formation was investigated. We found that cell spheroids formed in hydrogels that were exposed to MT were significantly smaller than spheroids formed without MT incubation, suggesting matrix stiffening played a crucial role in the sizes of cancer cell spheroids. Through utilizing highly specific and orthogonal enzymatic reactions, this hydrogel platform permits rapid and mild in situ cell encapsulation, as well as dynamic control of matrix stiffness for investigating the role of matrix stiffening on cell fate processes.
\end{abstract}

Keywords: Sortase, Mushroom Tyrosinase, Hydrogels, Extracellular Matrix Stiffening, Cancer 


\section{Introduction}

Hydrogels with dynamically tunable elastic moduli are increasingly being developed to mimic matrix stiffening and softening properties of the extracellular matrix (ECM). ${ }^{1-4}$ Biologically, tissue/matrix stiffening plays a significant role during tissue fibrosis and tumor progression. ${ }^{5,6}$ Therefore, designing hydrogels with temporally tunable stiffness will facilitate research concerning the influences of matrix stiffening on pathophysiological cell fate processes. Generally, dynamic hydrogels are designed to permit secondary crosslinking within the primary cell-laden hydrogel network. Utilizing this concept, Burdick and colleagues fabricated methacrylate-functionalized hyaluronic acid (MeHA) hydrogels via Michael-type addition reaction between methacrylates and thiols with off-stoichiometric ratio. ${ }^{7}$ Following initial crosslinking, gel stiffening was subsequently achieved by inducing light-and-radical-mediated chain growth polymerization of the remaining methacrylates. In another example, Suggs et al. utilized infrared radiation (IR) to stiffen alginatebased hydrogels loaded with temperature-sensitive liposomes containing calcium ions and gold nanorods. ${ }^{8}$ By heating the gold nanorods with IR, calcium ions were released from the disrupted liposomes, thus inducing additional crosslinking of alginate chains. Although this system could effectively control matrix elasticity both temporally and spatially, the heat generated from IR irradiation may not be ideal for certain applications. Gyarmati and Szilágyi demonstrated that timedependent disulfide bond formation could be used to induce secondary stiffening of hydrogels. ${ }^{9}$ Polymers containing excess free thiols undergo oxidation over time, leading to additional disulfide bonds and a stiffened matrix. However, this approach does not afford stringent control over the degree of gel stiffening. Disulfide exchange may also destabilize proteins critical to cell fate.

Anseth et al. showed that PEG-based hydrogels could be reversibly stiffened/softened by incorporating a photosensitive azobenzene crosslinker and irradiating with appropriate light. ${ }^{4}$ Hydrogel modulus was reversibly tuned ( 100-200 Pa) upon azobenzene cis-trans isomerization upon UV or visible light exposure. In addition to the use of UV light, another disadvantage was 
that the magnitude of stiffening was not physiologically relevant. Our lab has utilized host-guest interactions to provide physiologically relevant degree of reversible gel stiffening/softening, which were achieved by alternate addition and removal of macromolecular adamantane in cyclodextrin immobilized gels. ${ }^{10}$

In addition to the synthetic approaches described above, biological reactions (e.g., protein conformational changes, enzymatic reactions, etc.) have been utilized to modify matrix properties in cell-laden hydrogels. ${ }^{11-15}$ For example, Lutolf et al. prepared HA-based hydrogels by reacting HA-glutamine with PEG-Lysine in the presence of transglutaminase, ${ }^{16}$ which catalyzes the isopeptide bond formation between $\varepsilon$-amine of lysine and $\gamma$-carboxamide of glutamine. Two disadvantages of transglutaminase are its low substrate specificity (i.e., any lysine and glutamine residues) and calcium-dependency. Rocasalbas et al. showed that laccase, which catalyzes the formation of di-phenol crosslinks, could be used to crosslink phenolic-conjugated gelatin and chitosan. ${ }^{17}$ These gels, however, were formed in approximately two hours, rendering the system ineffectual for cell encapsulation. In an attempt to exploit enzymatic reactions for tuning hydrogel stiffness, our lab has demonstrated the use of mushroom tyrosinase (MT) to provide pathophysiologically relevant dynamic stiffening ( $\mathrm{G}^{\prime}$ from $0.5 \mathrm{kPa}$ to $4 \mathrm{kPa}$ ) of cell-laden PEGpeptide hydrogels. ${ }^{18}$ Specifically, we used MT to convert network-immobilized tyrosine residues into dihydroxyphenylalanine (DOPA) dimers, ${ }^{19}$ which led to in situ and on-demand matrix stiffening. This system was utilized to probe the effect of matrix stiffening on the activation of pancreatic stellate cells. ${ }^{18}$

Staphylococcus aureus sortase A (SrtA), a transpeptidase with small molecular weight ( 18-25 kDa) and high substrate specificity, has been widely used in $\mathrm{N}$ - and C-terminal labeling of proteins $^{20}{ }^{20}$ peptide cyclization, $^{21}$ and antibody modification. ${ }^{22,}{ }^{23}$ SrtA is a cysteine transpeptidase that ligates a short peptide sequence LPXTG (where $X$ is any amino acid but proline) and an oligoglycine nucleophile. ${ }^{24}$ Mechanistically, SrtA cleaves the amide bond between 
the threonine and glycine residues on LPXTG and forms a thiolacyl intermediate, followed by the release of a free glycine segment and the ligation of LPXT with an N-terminal oligolycine nucleophile (Fig. 1A). Notably, SrtA-mediated transpeptidation is reversible in the presence of additional enzyme and oligoglycine nucleophile. Recently, SrtA has been used to modify hydrogels. For example, Griffith and colleagues reported the use of SrtA for modifying biochemical properties of synthetic hydrogels and for recovering cells and proteins from gels crosslinked with SrtA peptide substrates. ${ }^{14,25}$

To expand the utility of enzyme-based crosslinking and tuning of hydrogel properties, we describe here the design of PEG-based hydrogels with orthogonal reactivity for both SrtA and MT. Specifically, we optimized SrtA-mediated transpeptidation for rapid crosslinking of PEG-peptide hydrogels with conditions suitable for in situ cell encapsulation. We then employed MT for postgelation in situ matrix stiffening. To afford orthogonal controls over gelation and stiffening, we synthesized two short tri-functional peptides (i.e., CYLPRTG and GGGGC). In addition to the sequences as SrtA substrates (i.e., LPRTG-amide and $\mathrm{NH}_{2}$-GGGG-amide), cysteine residues were added to permit facile thiol-ene photoclick reaction for conjugating the peptides to 8-arm PEG-norbornene (PEG8NB) ${ }^{26}$ Finally, the inclusion of tyrosine residues rendered the resulting gels susceptible to MT-triggered stiffening. We used SrtA $\mathrm{A}_{7 \mathrm{M}}$, a SrtA heptamutant (P94R, E105K, E108Q, D160N, D165A, K190E, K196T) with increased catalytic reactivity and independent of calcium, ${ }^{27}$ to fabricate the primary cell-laden hydrogel network. Subsequently, pendant tyrosine residues were dimerized upon on-demand incubation with MT. Lastly, we examined the cytocompatibility of the dual enzyme responsive gel system and investigated the effect of matrix stiffening on cell spheroid formation.

\section{Materials \& Methods}

\subsection{Materials}


8-arm PEG-OH (20 kDa) was obtained from JenKem Technology, USA. 5-norbornene-2carboxylic acid, N,N'-dicyclohexylcarbodiimide (DCC), 4-(dimethylamino)pyridine (DMAP) dimethyl phenylphosphonite, 2,4,6-trimethylbenzoyl chloride, lithium bromide, 2-butanone, and diethyl ether were purchased from Sigma-Aldrich. Fmoc-protected amino acids, $N, N, N^{\prime}, N^{\prime}-$ tetramethyl-O-(1H-benzotriazol-1-yl)uronium hexafluorophosphate $\quad$ (HBTU), and hydroxybenzotriazole (HOBt) were acquired from AnaSpec or ChemPep. N,N-dimethylformamide and dichloromethane were purchased from BDH Chemicals. BL21 E. Coli was purchased from New England Biolabs. Kanamycin sulfate was obtained from IBI Scientific. LB agar and broth were purchased from DOT Scientific. All other chemicals were obtained from Fisher Scientific unless otherwise noted.

\subsection{Macromer Synthesis}

Macromer 8-arm PEG-norbornene (PEG8NB, 20 kDa) was synthesized per established protocols with minor modifications. ${ }^{28}$ In brief, 5-norbornene-2-carboxylic acid (5-fold molar excess to hydroxyl group on PEG) was reacted with DCC (2.5x) in anhydrous DCM for 1 hour. The resulting product, norbornene anhydride, was transferred to an addition funnel and added drop wise to a two necked round bottom flask containing 8-arm PEG-OH, DMAP $(0.5 \mathrm{x})$, and pyridine (0.5x) under nitrogen. After overnight reaction on ice, the process was repeated to improve functionalization of norbornene to PEG. The PEG8NB product was precipitated in cold ethyl ether

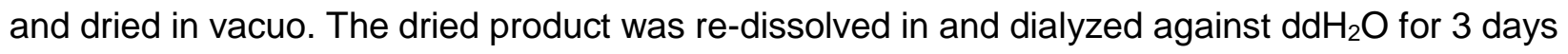
at room temperature (MWCO 6000-8000), followed by lyophilization. The degree of functionalization (ca. 80-90\%) was confirmed by ${ }^{1} \mathrm{H} \mathrm{NMR}\left(\mathrm{CDCl}_{3}, 500 \mathrm{MHz}\right.$, Bruker Advance 500): $\delta-(4.25 \quad m \quad 2 H), \quad 5.98 \quad(m \quad 1 H), \quad 6.23 \quad(m \quad 1 H)$ Photoinitiator lithium phenyl-2,4,6trimethylbenzoylphosphinate (LAP) was synthesized as described elsewhere. ${ }^{29}$

All peptides were synthesized with standard Fmoc coupling chemistry in an automated, microwave-assisted peptide synthesizer (Liberty 1, CEM). The crude peptides were cleaved (95\% 
TFA, $2.5 \% \mathrm{ddH}_{2} \mathrm{O}, 2.5 \% \mathrm{TIS}$, and $5 \%(\mathrm{w} / \mathrm{v})$ phenol) from resin for 3 hours at room temperature and precipitated in cold ethyl ether. The dried, crude peptides were purified using HPLC (Flexar System, Perkin Elmer). The purified peptides were confirmed by mass spectrometry (Agilent Technologies) and stored at $-20^{\circ} \mathrm{C}$.

PEG-peptide conjugates were prepared through reacting purified cysteine-bearing peptides with PEG8NB via thiol-norbornene photoclick chemistry. Peptides were dissolved in PBS, and Ellman's assay was performed to determine the concentration of cysteine-bearing peptide in solution. The peptides (1.5-fold excess to norbornene groups) were added to a flask containing PEG8NB and photoinitiator LAP (5 mM). The mixture was subjected to light (Omnicure s1000, $\left.365 \mathrm{~nm}, 40 \mathrm{~mW} / \mathrm{cm}^{2}\right)$ for 30 minutes. At 15 minutes, additional LAP ( $5 \mathrm{mM}$ ) was added to improve conjugation efficiency. The PEG-peptide products were dialyzed against $d_{d d} \mathrm{H}_{2} \mathrm{O}$ for 2 days to remove excess peptides, lyophilized, and stored at $-20^{\circ} \mathrm{C}$.

\subsection{Heptamutant Sortase A Expression and Purification}

$\mathrm{Ca}^{2+}$-independent, heptamutant sortase A (P94R, E105K, E108Q, D160N, D165A, K190E, K196T) was expressed as described previously. ${ }^{30}$ In brief, competent BL21 E. Coli cells were transformed with pet30b-7M SrtA plasmid (a gift from Hidde Ploegh. Addgene plasmid \#51141). The transformed cells were grown on an LB-agar selection plate containing kanamycin (30 $\mu \mathrm{g} / \mathrm{mL}$ ). An individual colony from the selection plate was picked out and inoculated (220 rpm at $\left.37^{\circ} \mathrm{C}\right)$ overnight in $10 \mathrm{~mL}$ LB media supplemented with kanamycin $(30 \mu \mathrm{g} / \mathrm{mL})$. The culture was diluted to $1 \mathrm{~L} \mathrm{LB}$ media supplemented with kanamycin and shaken at $37^{\circ} \mathrm{C}$ until the $\mathrm{OD}_{600}$ reached 0.4-0.8 ( 4 hours). Following induction of SrtA $_{7 \mathrm{M}}$ expression by IPTG $(400 \mu \mathrm{M})$ for 3 hours, cells were isolated by centrifugation (8000 rpm, 15 minutes) and stored at $-80^{\circ} \mathrm{C}$ prior to lysis. Cells were lysed by incubating the pellets in lysis buffer $(20 \mathrm{mM}$ Tris, $50 \mathrm{mM} \mathrm{NaCl}, 0.2 \mathrm{mg} / \mathrm{mL}$ lysozyme, $1 \mathrm{mM}$ PMSF, 1x Halt ${ }^{\mathrm{TM}}$ EDTA-free protease inhibitor Cocktail, and DNase I) for 30 minutes followed by sonication ( 2 cycles of 3 minutes with $30 \%$ duty cycle, $20 \%$ amplitude followed by a 
3 minute cool-down). Lysates were cleared by centrifugation for 20 minutes at $10,000 \times \mathrm{g}$ at $4^{\circ} \mathrm{C}$ and purified with His60 Ni Superflow resin and columns per manufacturer's protocol. The purity of the eluted fractions was analyzed by SDS-PAGE and Western Blot with primary antibody against 6 xhistidine tag. Pure fractions were combined and concentrated to $\sim 50 \mathrm{mg} / \mathrm{mL}$ with Ultra15 Centrifugal Filter Units (Amicon). Buffer exchange was performed against DPBS with 7k MWCO Zeba Spin desalting columns (Thermo Fisher Scientific). Concentrated, desalted SrtA $\mathrm{A}_{7 \mathrm{M}}$ aliquots were sterile filtered and stored at $-80^{\circ} \mathrm{C}$. Final $\mathrm{SrtA}_{7 \mathrm{M}}$ concentration was determined using Ellman's assay (SrtA $A_{7 M}$ contains one free cysteine). SrtA $A_{7 M}$ activity was verified against SrtA $A_{W T}$ utilizing the Sensolyte 520 Sortase A activity assay kit (Anaspec Inc.) per the manufacturer's protocol.

\subsection{Sortase A-mediated Hydrogel Crosslinking and Characterization}

Hydrogels were fabricated from conjugating PEG-YLPRTG and $\mathrm{NH}_{2}$-GGGG-PEG with specified concentrations of $\mathrm{SrtA}_{7 \mathrm{M}}$. Both PEG-peptide macromers were dissolved in PBS and combined. Gelation was initiated by adding $\mathrm{SrtA}_{7 \mathrm{M}}$ and then proceeded for 10 minutes unless otherwise specified. The gels were allowed to swell in PBS at $37^{\circ} \mathrm{C}$ for 16 hours before measuring the hydrogel storage and loss moduli (G' \& G") with oscillatory rheometry in strain-sweep mode (CVO 100, Bohlin). The reported G' were averaged from the linear region of the modulus-strain curves. In situ rheometry was performed in time sweep mode (0.5\% strain, $1 \mathrm{~Hz}$ frequency, and $90 \mu \mathrm{m}$ gap size at $25^{\circ} \mathrm{C}$ ). Immediately after adding $\mathrm{SrtA}_{7 \mathrm{M}}$, the precursor solution was mixed and pipetted between the rheometer platform and $8 \mathrm{~mm}$ parallel plate geometry. To prevent drying, the rim of the geometry was sealed with mineral oil.

\subsection{MT Stiffening and Characterization}

Hydrogels were fabricated and incubated in PBS for 16 hours. Using oscillatory rheometry in strain-sweep mode as described above, moduli were measured prior to and after hydrogels were incubated with $\mathrm{MT}(1 \mathrm{kU} / \mathrm{mL})$ at $37^{\circ} \mathrm{C}$. Due to volumetric shrinkage following MT-triggered 
stiffening, the gap sizes for $\mathrm{R}_{\mathrm{GGGG}: \mathrm{LPRTG}}=1, \mathrm{R}_{\mathrm{GGGG}: \mathrm{LPRTG}}=3$ were reduced from $525 \mu \mathrm{m}$ to $475 \mu \mathrm{m}$ and $700 \mu \mathrm{m}$ to $625 \mu \mathrm{m}$, respectively.

\subsection{MIN6 and COLO-357 Cell Culture}

MIN6 cells, a mouse insulinoma cell line (a gift from Prof. Raghu Mirmira at IU School of Medicine), were maintained in high glucose DMEM (HyClone) containing 10\% FBS (Gibco), 1xAntibiotic-Antimycotic (Invitrogen, $100 \mathrm{u} / \mathrm{mL}$ penicillin, $10 \mu \mathrm{g} / \mathrm{mL}$ streptomycin, and $250 \mathrm{ng} / \mathrm{mL}$ Fungizone), and $50 \mu \mathrm{M} \beta$-mercaptoethanol. COLO-357 cells, a pancreatic adenocarcinoma cell line (a gift from Prof. Murray Korc at IU School of Medicine), were maintained in high glucose DMEM containing 10\% FBS, and 1x-Antibiotic-Antimycotic. For both cell lines, media was refreshed every 2-3 days.

\subsection{SrtA $\mathrm{A}_{7 \mathrm{M}}-$ mediated Encapsulation of MIN6, and Characterization of Cell Viability and Morphology}

Prior to encapsulation, cells were trypsinized, counted (Countess ${ }^{\circledR}$ II Automated Cell Counter, ThermoFisher), and re-suspended in Hank's Balanced Salt Solution (HBSS). Resuspended cells were mixed with sterile-filtered pre-polymer solution containing desired concentrations of PEG-YLPRTG and $\mathrm{NH}_{2}$-GGGG-PEG. Gelation was initiated by adding SrtA $\mathrm{A}_{7 \mathrm{M}}$ at desired concentration. The cell-polymer solution was immediately transferred to a $1 \mathrm{~mL}$ syringe mold and cured for 10 minutes to ensure completion prior to placing the hydrogels in fresh media. Metabolic activity was monitored with Alamarblue ${ }^{\circledR}$ reagent. In brief, cell-laden gels were transferred to Alamarblue ${ }^{\circledR}$ reagent (diluted 10-fold with cell culture media) and incubated for 16 hours. Following incubation, $200 \mu \mathrm{L}$ of the diluted media was transferred to a clear 96 -well plate and the fluorescence was determined by a microplate reader (SynergyHT, Biotek: ex $560 \mathrm{~nm}$ em $590 \mathrm{~nm}$ ). Additionally, a LIVE/DEAD ${ }^{\circledR}$ staining kit (Life Technologies) was used to assess viability. Per the manufacturer's staining protocol, the hydrogels were placed in PBS diluted with calcein AM and an ethidium homodimer and stained for 1 hour. Gels were washed in fresh PBS to remove 
excess dye, and imaged with a confocal microscope (Olympus Fluoview FV100 laser scanning microscope).

\subsection{In Situ MT Stiffening of COLO-357-laden Hydrogels and Characterization of Cell Viability and Morphology}

Cell-laden hydrogels were fabricated using $\mathrm{SrtA}_{7 \mathrm{M}}$ as described above. To induce secondary stiffening, the cell-laden hydrogels were transferred to a 24-well plate containing media supplemented with $1 \mathrm{kU} / \mathrm{mL} \mathrm{MT}$ and incubated for 6 hrs at $37^{\circ} \mathrm{C}$ in $5 \% \mathrm{CO}_{2}$. The hydrogels were transferred to and maintained in a new well containing fresh media. Live/dead staining and imaging were performed as described above, and the spheroid diameter was measured using Nikon NIS-element software.

\subsection{Statistical Analysis}

Statistical analysis was conducted using a two-tailed t-test or a two-way analysis of variance (ANOVA) followed by a Bonferroni's post-hoc test. A p value $<0.05$ was considered statistically significant. Single, double, and triple asterisks represent $p<0.05$ and 0.01 , and 0.001 , respectively. All studies were performed independently for at least three times and the quantitative results were presented are mean \pm SEM.

\section{Results}

\subsection{Expression of SrtA $\mathrm{A}_{\mathrm{M}}$}

We used heptamutant SrtA (i.e., SrtA $\mathrm{A}_{7 \mathrm{M}}$ ) in this study for its improved stability and enhanced reactivity compared with wild type SrtA (i.e., SrtAWT). ${ }^{31}$ Successful expression and purification of $\mathrm{SrtA}_{7 \mathrm{M}}(\mathrm{MW}$ : $18 \mathrm{kDa}$ ) was confirmed by SDS-PAGE (Fig. 1B) and Western Blot with antibody against 6 xhistidine tag (data not shown). The catalytic activity of SrtA 7 mas verified first by comparing enzymatic activity against the commercially available Srt ${ }_{W T}$ in the presence of $\mathrm{Ca}^{2+}$. While both enzymes were active in a $\mathrm{Ca}^{2+}$ rich environment (Fig. 1C), SrtA $\mathrm{A}_{7 \mathrm{M}}$ exhibited 
higher catalytic activity than SrtAwt. In a $\mathrm{Ca}^{2+}$ deprived environment (Fig. 1D), SrtAwt completely lost its catalytic activity whereas $\mathrm{SrtA}_{7 \mathrm{M}}$ was still active. These data confirm the $\mathrm{Ca}^{2+}$-independent catalytic activity of $\mathrm{SrtA}_{7 \mathrm{M}}$ as described in the literature..$^{27,31,32}$

\subsection{SrtA $\mathrm{A}_{7 \mathrm{M}}$-mediated crosslinking of PEG-peptide hydrogels}

In order to exploit $\mathrm{SrtA}_{7 \mathrm{M}}$ for hydrogel crosslinking, we prepared PEG-peptide conjugates by tethering SrtA substrates (GGGG or LPRTG) to PEG8NB macromers via thiol-norbornene reaction (Fig. 2A). Purified peptides bearing cysteine and tyrosine residues $\left(\mathrm{NH}_{2}\right.$-GGGGC-amide or $\mathrm{NH}_{2}$-CYLPRTG-amide) were used to permit thiol-norbornene coupling of the PEG-peptide conjugates, which were susceptible to both $\mathrm{SrtA}_{7 \mathrm{M}}$ mediated gel crosslinking and MT-triggered stiffening. In situ rheometry was used to determine gelation efficiency at varied concentrations of $\mathrm{SrtA}_{7 \mathrm{M}}$. At a stoichiometric molar ratio of GGGG:LPRTG $\left(\mathrm{R}_{\mathrm{GGGG}} \mathrm{LPRTG}=1\right)$ and total PEG-peptide concentration of $2 \mathrm{wt} \%$ (i.e., $1 \mathrm{wt} \%$ PEG-LPRTG and $1 \mathrm{wt} \% \mathrm{NH}_{2}$-GGGG-PEG), the gelation was accelerated at higher concentrations of $\mathrm{SrtA}_{7 \mathrm{M}}$, as indicated by the rapid increases in shear modulus (G', Fig. 2B). As expected, the speed of gelation scaled with $\mathrm{SrtA}_{7 \mathrm{M}}$ concentration. Next, we investigated the effect of $\mathrm{Srt}_{7 \mathrm{M}}$ concentration on the elastic modulus at a fixed total concentration of PEG-peptide conjugates (3 wt\%, $\mathrm{R}_{\mathrm{GGGG}}$ LPRTG=1, Fig. 2C). Gelation was allowed to continue for 10 minutes prior to placing the gels in buffer for equilibration (16 hours). Fig. 2C shows that $\mathrm{SrtA}_{7 \mathrm{M}}$ concentration did not significantly affect the final moduli of the hydrogels. We further determined that $300 \mu \mathrm{M} \mathrm{SrtA}_{7 \mathrm{M}}$ treatment for 10 minutes was sufficient for complete gelation as extending gelation time to 960 minutes only slightly but not statistically increased gel moduli $(\sim 5,700, \sim 6,350$, and $\sim 6,500 \mathrm{~Pa}$ for 10,60 , and 960 minutes gelation, respectively. Fig. 2D). Finally, the moduli of the hydrogels could be tuned by increasing the total PEG-peptide concentration. Specifically, the moduli for $2 \%, 3 \%$, and $4 \%$ of PEG-peptide conjugates were $\sim 2,700, \sim 6,000, \sim 10,000 \mathrm{~Pa}$, respectively (Fig. 2E). 


\subsection{Cytocompatibility of SrtA ${ }_{7 M}$-crosslinked hydrogels}

To evaluate the cytocompatibility of SrtA $_{7 \mathrm{M}}$-crosslinked PEG-peptide hydrogels, we encapsulated MIN6 $\beta$-cells, a particularly vulnerable cell type when encapsulating in bio-inert hydrogels at low cell density $\left(<10^{6}\right.$ cells $\left./ \mathrm{mL}\right) \cdot{ }^{33}, 34$ Similar to results previously reported in alternative crosslinking systems, ${ }^{35}$ the encapsulated cells exhibited increasing metabolic activity over the course of the experiment (Fig. 3A). Additionally, live/dead staining images showed that virtually all cells remained alive following encapsulation (day 1) and formed spheroids in the hydrogels after 10 days of in vitro culture.

\subsection{Dynamic MT stiffening of SrtA7M-crosslinked PEG-peptide hydrogels}

We conferred MT-sensitivity to the $\mathrm{SrtA}_{7 \mathrm{M}}$-crosslinked hydrogels by simply incorporating one tyrosine residue in the peptide crosslinker (Fig. 4A). The primary gel crosslinking was achieved by $300 \mu \mathrm{M}$ SrtA $_{7 M}$ treatment of PEG-peptide conjugates. The equilibrated gels were placed in buffer containing MT $(1 \mathrm{kU} / \mathrm{mL})$ for 3 to 6 hours. As shown in Figure $4 \mathbf{B}$, the moduli of hydrogels increased $\sim 1.25$-fold and $\sim 1.5$-fold after 3 and 6 hours of MT incubation. Secondary crosslinking via di-tyrosine formation was evident from the emergence of yellow/brown color in the MT-treated gels (Fig. 4C). Specifically, the hydrogels were transparent prior to MT incubation but turned yellow and brown after 3 and 6 hours of MT treatment. Additionally, the hydrogels underwent volumetric shrinkage and $-25 \%$ reduction in diameter after 6 hours of MT incubation. These results indicate that inserting a single tyrosine residue into the peptide crosslinker successfully rendered the PEG-peptide hydrogels susceptible to not only SrtA $\mathrm{A}_{7 \mathrm{M}}$-mediated crosslinking but also MT-triggered in situ stiffening.

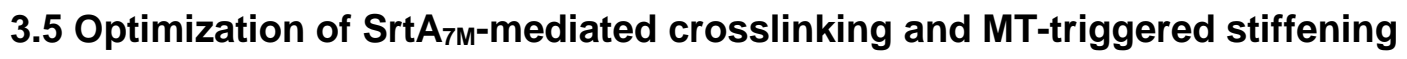

We varied the substrate ratio (i.e., $\mathrm{R}_{\mathrm{GGGG}} \mathrm{YLPRTG}$ ) at a fixed enzyme concentration so as to determine the effect of the substrate concentrations on hydrogel crosslinking. First, hydrogels 
were fabricated per the formulations in Table 1 with a fixed LPRTG concentration and increasing concentrations of GGGG. Intriguingly, PEG-peptide hydrogels containing an equimolar ratio of peptide substrates exhibited substantially higher moduli ( $\mathrm{G}^{\prime}: \sim 10,000 \mathrm{~Pa}$ ) than the gels fabricated at an $\mathrm{R}_{\mathrm{GGGG}: \mathrm{YLPRTG}}=1 / 3\left(\mathrm{G}^{\prime} \sim 1,000 \mathrm{~Pa}\right)$ and $3\left(\mathrm{G}^{\prime}: \sim 1,500 \mathrm{~Pa}\right.$. Fig. 5A). Furthermore, hydrogels containing increasing amounts of the nucleophilic oligoglycine (i.e., GGGG) exhibited significantly higher gel fraction $(0.4,0.7$, and 0.8 for $\mathrm{R}=1 / 3,1$, and 3 , respectively). Because of its higher gel fraction, we investigated the effect of MT-triggered stiffening on hydrogels fabricated with $\mathrm{R}_{\text {GGGG:YLPRTG }}=3$ at two different PEG-peptide concentrations (Fig. 5C). Surprisingly, hydrogels composed of 6 wt\% and 10 wt\% PEG-peptide conjugates (Table 1) exhibited similar initial shear moduli of $\sim 1,000 \mathrm{~Pa}$. Upon incubation with MT for 24 hours, the moduli of the $6 \mathrm{wt} \%$ hydrogels increased to $\sim 2,000 \mathrm{~Pa}$ (approximately 2-fold), whereas that of the $10 \mathrm{wt} \%$ gels increased to $\sim 3,000 \mathrm{~Pa}$ (approximately 3-fold) due to an increased concentration of the tyrosine residue (Table

1).

Table 1. Formulations of PEG-peptide hydrogels used in Figure 5.

\begin{tabular}{|c|c|c|c|c|c|}
\hline RGGGG:YLPRTG & $\begin{array}{c}\text { PEG-YLPRTG } \\
(\mathbf{w t} \%)\end{array}$ & $\begin{array}{c}\mathbf{N H}_{\mathbf{2}} \text {-GGGG-PEG } \\
\mathbf{( w t \% )}\end{array}$ & $\begin{array}{c}\text { Total PEG } \\
\mathbf{( w t \% )}\end{array}$ & $\begin{array}{c}\text { [YLPRTG] } \\
\mathbf{( m M )}\end{array}$ & $\begin{array}{c}\text { [GGGG] } \\
(\mathbf{m M})\end{array}$ \\
\hline $1 / 3$ & 1.5 & 0.5 & 2 & 6 & 2 \\
\hline 1 & 1.5 & 1.5 & 3 & 6 & 6 \\
\hline 3 & 1.5 & 4.5 & 6 & 6 & 18 \\
\hline 3 & 2.5 & 7.5 & 10 & 10 & 30 \\
\hline
\end{tabular}

\subsection{Effect of MT-triggered stiffening on COLO-357 cell viability and spheroid formation}

Owing to its desirable gel fraction and degree of stiffening, we utilized 10 wt $\%$ hydrogels with an RGGGG:YLPRTG $=3$ to encapsulate COLO-357 cells, a pancreatic cancer cell line, to investigate the role of dynamic matrix stiffening on cell spheroid formation and morphology. The hydrogels were maintained in media and stiffened via MT incubation on D1 post-encapsulation. Live/dead staining results show that nearly all cells survived the encapsulation process. By D10, 
the cells grew into multi-cell spheroids, even though the gels contained no bioactive motif and were not proteolytically labile (Fig. 6A). Spheroids formed in gels without MT-triggered stiffening were significantly $(p<0.01)$ smaller than those formed in gels with MT-triggered stiffening (Fig. 6B).

\section{Discussion}

Three-dimensional matrices capable of mimicking pathophysiologically relevant ECM stiffening are actively developed for tissue engineering applications. Although many approaches have been utilized successfully for in vitro cell studies designed to understand the role of matrix stiffening on cell fate processes, most require chemical additives, light, heat, and/or do not provide stringent control over the rate and magnitude of stiffening. In contrast, enzymatic approaches to fabricating and modifying hydrogels are highly specific, predictable, and can occur under mild reaction conditions. In this contribution, we explored the use of two enzymes (i.e., SrtA $A_{7 M}$ and MT) with orthogonal reactivity for initial crosslinking and subsequent tuning of PEG-based hydrogel properties. PEG-based hydrogels are advantageous due to the high permeability of the network, which is critical for facile exchange of nutrients and cellular metabolic wastes. ${ }^{36,37}$ Additionally, PEG-based hydrogels can be rendered bioactive through the conjugation of short peptide motifs, including cell adhesion peptides (e.g. RGDS, YIGSR, etc.) and MMP-cleavable sequences, both of which are critical in promoting cell survival and matrix remodeling. ${ }^{38-40}$

We rendered the hydrogels susceptible to sequential SrtA ${ }_{7 M}-$ mediated crosslinking (Fig. 2A) and MT stiffening (Fig. 4A) by tethering a single set of peptide substrates (i.e., YLPRTG and GGGG) to PEG8NB macromers. SrtA is a bacterial enzyme with high specificity of peptide substrates that are absent in nearly all mammalian proteins. ${ }^{25}$ As a result, the use of SrtA should not adversely affect the structure and function of proteins in mammalian cell culture. SrtA and its various mutated versions have been increasingly used in bioconjugation chemistry, ${ }^{23,30}$ live cell labeling, ${ }^{41}$ and more recently in hydrogel-related applications. ${ }^{14,}{ }^{25}$ However, the optimization of 
SrtA-based transpeptidation in hydrogel fabrication has not been reported. SrtA $\mathrm{A}_{7 \mathrm{M}}$ was used here as its mutations (P94R/D160N/D165A/K190E/K196T) increased catalytic efficiency $\left(k_{\text {cat }} / K_{m}\right)$ by $140-$ fold $)^{42}$ and conferred calcium independency (E105K/E108A) due to the stabilization of the critical calcium-binding $\beta 6 / \beta 7$ loop. ${ }^{31}$ We confirmed the $S r t A_{7 M}$ expressed for this study was active (Fig. 1B) and exhibited high catalytic activity even in $\mathrm{Ca}^{2+}$ deprived solution (Fig. 1C).

We optimized the formulations and crosslinking conditions of $\mathrm{Srt}_{7 \mathrm{M}}$-mediated primary gel crosslinking and characterized MT-catalyzed reactions to obtain a pathophysiologically relevant degree of stiffening. Initially, we determined the gelation kinetics with different concentrations of enzyme and an equimolar ratio of the peptide substrates (Fig. 2B). As expected, gelation time was shorter at higher enzyme concentrations due to an increase in the maximum velocity $\left(\mathrm{V}_{\max }\right)$ of the transpeptidation reaction. We further characterized the enzymatic crosslinking by establishing that gelation could be achieved at low concentrations of $\operatorname{SrtA}_{7 \mathrm{M}}(150 \mu \mathrm{M})$ (Fig. 2C) and a rapid gelation time of 10 minutes (Fig. 2D). Although complete gelation could be achieved in 10 minutes at concentrations as low as $150 \mu \mathrm{M}$ of $\mathrm{SrtA}_{7 \mathrm{M}}$, we utilized $300 \mu \mathrm{M}$ of $\mathrm{Srt}_{7 \mathrm{M}}$ for its adequate gel point ( $\sim 2$ minutes) suitable for pipetting, solution mixing, and cell encapsulation. Note that the crosslinking density of the hydrogels was highly tunable through adjusting the total concentration of the PEG-peptide macromers (Fig. 2E).

In situ encapsulation of MIN6 $\beta$-cells was used to demonstrate the cytocompatibility of the SrtA $A_{7 M}-$ mediated gel crosslinking. MIN6 cells were used because of their sensitivity to cellular damages induced by radical-based gel crosslinking. ${ }^{33,34}$ The encapsulated cells were alive, and proliferated into multicellular spheroids (Fig. 3). This can be attributed to the high substrate specificity of the enzymatic reaction, as well as the mild reaction conditions during encapsulation. We postulate that $\mathrm{SrtA}_{7 \mathrm{M}}$ can be utilized for encapsulation of a host of mammalian cell lines due to these advantages. Although not demonstrated in this work, bioactive moieties, such as RGDS or MMP-sensitive peptides, could be incorporated into the hydrogel network using SrtA $\mathrm{Am}_{7 \mathrm{M}}$ transpeptidation, to further enhance survival and function of the cells. 
Our lab has previously established that the degree of stiffening could be directly controlled by adjusting MT incubation time and the number of tyrosine residues on a peptide crosslinker. ${ }^{18}$ Here we conferred susceptibility of the SrtA-crosslinked hydrogel network to MT secondary stiffening by simply incorporating a tyrosine residue on the peptide crosslinker (Fig. 4A). The dityrosine formed within the SrtA $\mathrm{A}_{7 \mathrm{M}}$-mediated crosslinked hydrogel increased the crosslinking density of the hydrogel and hence stiffened the hydrogel (Fig. 4B). The conversion of tyrosine residues to di-tyrosine also leads to a color change from transparent to brown further qualitatively confirming MT stiffening of the hydrogels (Fig. 4C). As established here, stiffening could be achieved "on demand" when the hydrogels are placed in solution containing tyrosinase. Additionally, the degree of stiffening could be increased by simply incorporating additional residues in the peptide substrates (i.e. YLPRTG and GGGGY); however, our results suggest that the incorporation of a single tyrosine residue is sufficient for inducing pathophysiologically relevant magnitudes of stiffening.

The inherent reversibility of the transpeptidation reaction is a notable drawback of utilizing $\mathrm{SrtA}_{7 \mathrm{M}}$. This is due the cleaved glycine-containing by-products also serve as substrates for additional transpeptidation. ${ }^{43}$ In solution with low glycine content, the thioacyl LPXT-SrtA ${ }_{7 M}$ intermediate can be hydrolyzed rendering the substrate unreactive. ${ }^{43}$ To mitigate these potentially undesirable side reactions, researchers often employ excess oligoglycine to the LPXTG substrate or have designed substrates with unreactive cleavage byproducts. ${ }^{44-46}$ We sought to investigate SrtA $_{7 \mathrm{M}}$-crosslinking in the presence of different ratios of the substrates, PEG-LPRTG and PEGGGGG (Figs. 5A, B) and found that the concentration of the oligoglycine substrate plays a significant role in determining the mechanical properties of the hydrogels. Hydrogels fabricated with $R_{G G G G: L P R T G}=1$ yielded a significantly stiffer matrix suggesting that an equimolar ratio of the substrates yielded a hydrogel network with less structural defects. The softer hydrogel at low concentrations of GGGG ( R $_{G G G G: Y L P R T G}=1 / 3$ ) also exhibited a lower gel fraction (Figs. 5A, B) likely due to the loss of unreacted PEG-LPRTG macromer. While a three-fold excess of GGGG 
$\left(R_{G G G G: Y L P R T G}=3\right)$ also produced a softer matrix, the gel fraction was comparable to the gels formed at stoichiometric ratio (Figs. 5A, B). We speculate that an excess of unreacted PEGGGGG arms created network defects similar to the hydrogels fabricated with $\mathrm{R}_{\mathrm{GGGG}} \mathrm{YLPRTG}=1 / 3$; however, additional oligoglycine substrate drove the reaction towards transpeptidation, hence yielding an increased gel fraction. Due to its higher gel fraction, we explored the effect of MT stiffening on hydrogels with $\mathrm{R}_{\mathrm{GGGG}}$ YLPRTG $=3$ at different total concentrations of PEG (Fig. 5C). At both 6 wt $\%$ and 10 wt $\%$ of total PEG-peptide concentrations, the initial shear modulus was similar. We postulate that at constant $V_{\max }$ for both conditions, additional time is required for $\mathrm{SrtA}_{7 \mathrm{M}}$ to consume the substrate and therefore to reach complete gelation for the $10 \mathrm{wt} \%$ hydrogels. Moreover, we observed a greater degree of stiffening in the $10 \mathrm{wt} \%$ hydrogels due to a higher concentration of tyrosine residues (Fig. 5C).

Future investigations are required to further elucidate the role of the substrate ratio on SrtA $_{7 M}$ reactions and hydrogel crosslinking kinetics. However, due to its desirable gel fraction, initial modulus, and physiologically relevant magnitude of stiffening (from $\sim 1$ to $3 \mathrm{kPa}$, Fig. $5 \mathrm{C}$ ), we utilized $10 \mathrm{wt} \%$ hydrogels with $\mathrm{R}_{\mathrm{GGGG}: Y L P R T G}=3$ for encapsulation of pancreatic cancer cells to investigate the effect of MT stiffening on cell spheroid formation. The color change, which did not interfere with confocal imaging, confirmed secondary stiffening of cell-laden hydrogels. Note that these gels were crosslinked without protease sensitive motifs, thus enabling the study of dynamic matrix stiffening on cell spheroids formation without the complication of cell-mediated proteolytic gel degradation. Clearly, stiffening of the hydrogels inhibited cell proliferation as indicated by the smaller spheroids (Figure 6). This is consistent with our previous report exploring the effect of matrix stiffness on COLO-357 cell spheroid sizes. ${ }^{47}$ This can be attributed to the increased density of the polymer network. Notably, both initial crosslinking and subsequent stiffening occurred without causing cell damage. While the MT-triggered stiffening process were performed for only 6 hours, the duration by which gels were stiffened could be extended to multiple days to closely mimic in vivo stiffening of tissues. This demonstrates the efficacy of the dual enzymatic 
crosslinking strategy for controlling the morphology of pancreatic cancer cells. Moreover, this system could be utilized to generate spheroids of various cell types in 3D. The inherent reversibility of $\mathrm{SrtA}_{7 \mathrm{M}}$ transpeptidation should allow for site specific degradation of the hydrogels. ${ }^{25}$ Therefore, future experiments may be focused on generation and recovery of tumor spheroids for further functional and biological characterizations. Although protease sensitive crosslinkers were not incorporated into the hydrogels in the current study, future work may include the addition of bioactive peptides to allow for cell-driven degradation. For example, a peptide linker could be synthesized with NH2-GGG followed by a protease-sensitive sequence, such as matrix metalloproteinase (MMP) cleavable peptide GPQGIWGQ. MMP-sensitive sequence could also be placed before LPRTG. After SrtA-mediated gelation, hydrogel crosslinks would become PEGLPRTGGGGPQGIWGQ-PEG or PEG-GPQGIWGQLPRTGGG-PEG, both of which will be susceptible to MMP-mediated cleavage.

\section{Conclusion}

In summary, we have developed a purely enzymatic strategy for hydrogel crosslinking and on-demand stiffening. The specificity of the substrates allows for rapid Srt $_{7 \mathrm{M}}$-mediated crosslinking, whereas the incorporation of a single tyrosine residue into the peptide crosslinker warrants highly controllable hydrogel stiffening. Due to the specificity of the substrates as well as the mild reaction conditions, $\mathrm{SrtA}_{7 \mathrm{M}}$-mediated crosslinking was highly cytocompatible even for a sensitive cell type (i.e., MIN6). The crosslinking and subsequent stiffening of the hydrogels could be optimized by adjusting the concentrations of the PEG-peptides. Finally, stiffening of cell-laden hydrogels inhibited cell spreading effectively controlling the formation and morphology of cell spheroids. Future work will include further optimization of the $\mathrm{SrtA}_{7 \mathrm{M}}$ crosslinking strategy as well

as exploiting this system for investigating the underlying mechanisms of cellular response to a stiffening microenvironment. 


\section{Acknowledgment}

This project was funded in part by an NSF CAREER Award (DMR \#1452390) and the Department of Biomedical Engineering at IUPUI. The authors thank Dr. Tsai-Yu Lin for his assistance with SrtA $_{7 \mathrm{M}}$ expression, purification and characterization, as well as Mr. John Bragg for his assistance in peptide synthesis. 
A.

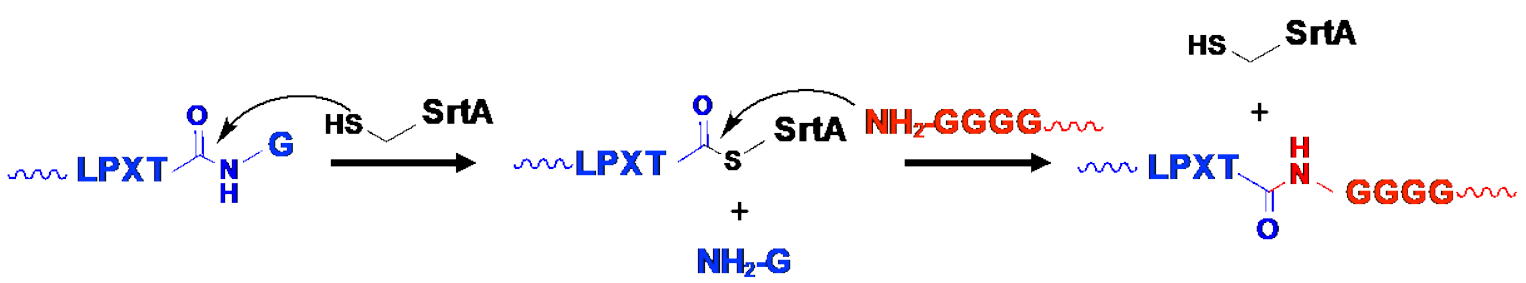

B.

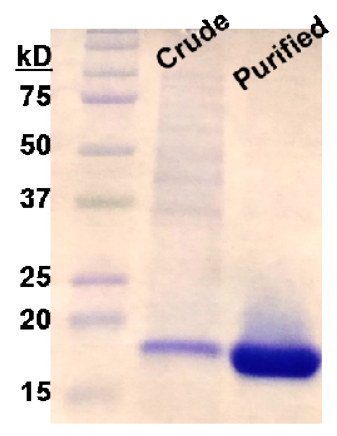

C.

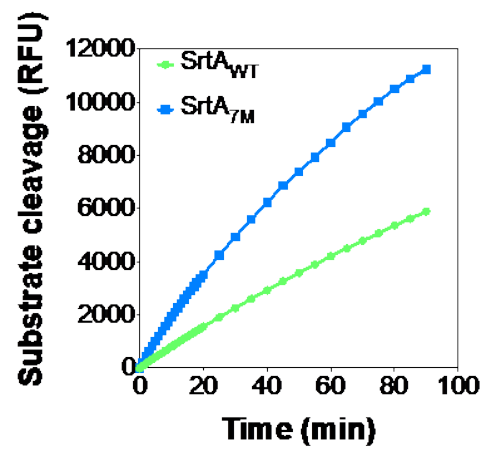

D.

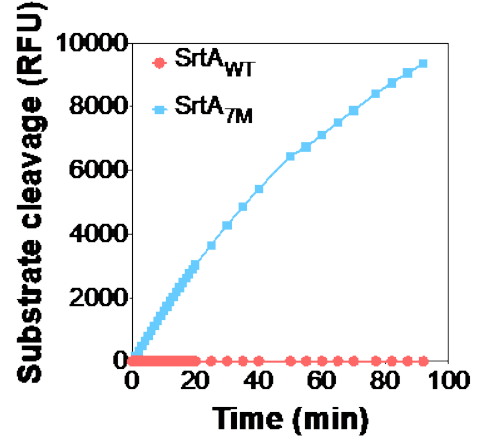

Figure 1. (A) Mechanism of SrtA-mediated transpeptidation. Cysteine residue at the active center of SrtA recognizes the amide bond between $T$ and $G$ residues of the conserved LPXTG motif, forming a thioacyl intermediate. The intermediate is resolved following the attack of an amino oligoglycine nucleophile, yielding the final product (i.e., LPXTGGGG) and regenerating SrtA. (B) SDS-PAGE of SrtA $\mathrm{A}_{7 M}(-18 \mathrm{kDa})$ expressed in BL21 E. Coli. SrtA ${ }_{w T}$ and SrtA $\mathrm{Am}_{7 \mathrm{M}}$ activity in Tris-HCl $(\mathrm{pH}=8.0)$ with $50 \mathrm{nM}$ SrtA, $2 \mu \mathrm{M}$ 5-FAM/QXL FRET peptide substrate, and $2 \mathrm{mM}$ of oligo-glycine peptide (i.e., GGGGC) in the presence (C) or absence (D) of $10 \mathrm{mM} \mathrm{CaCl}_{2}$. SrtA induced substrate cleavage was detected by fluorescence of the cleaved 5-FAM substrate (excitation/emission = 490/520). RFU: relative fluorescence units. 
A.
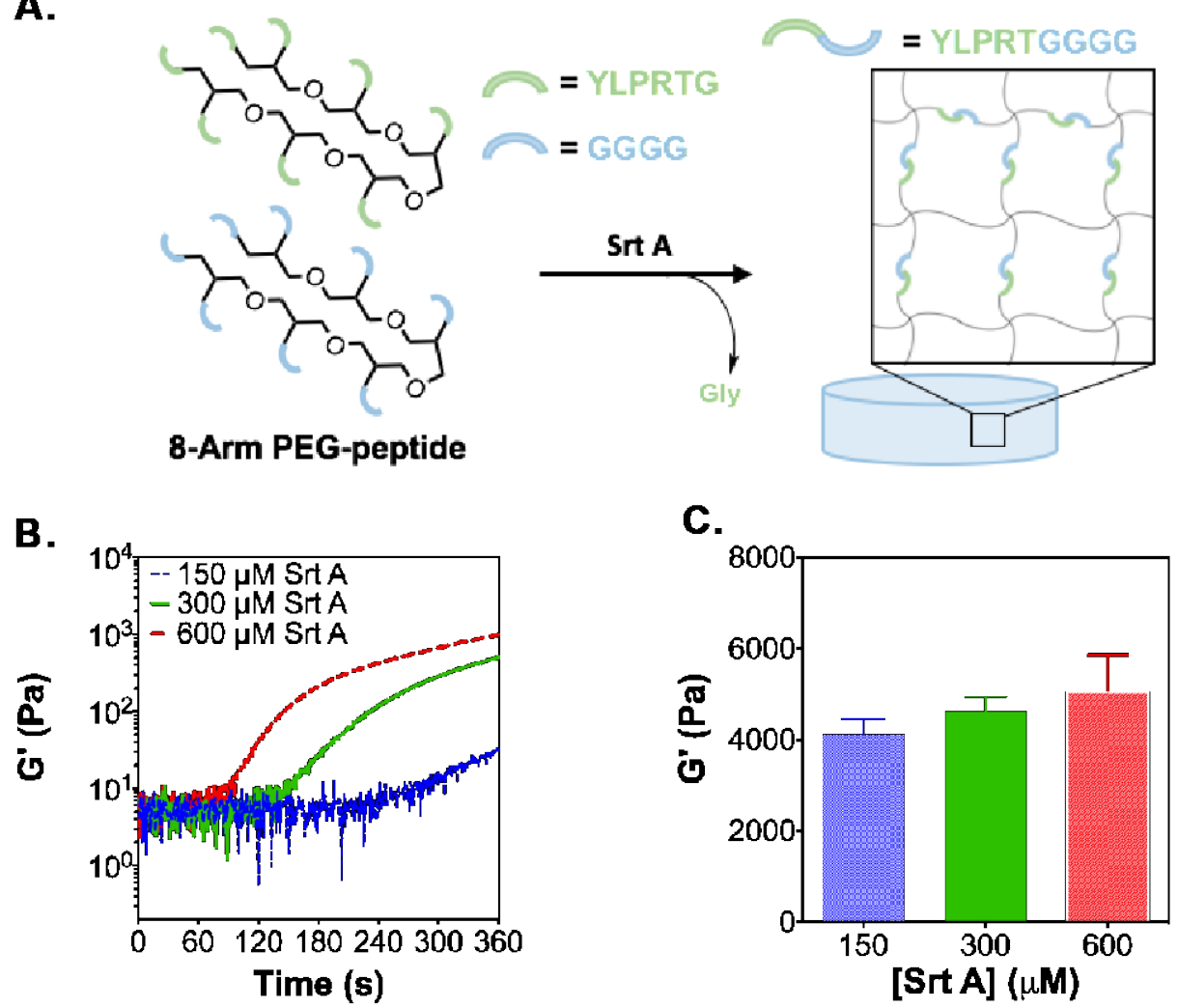

D.

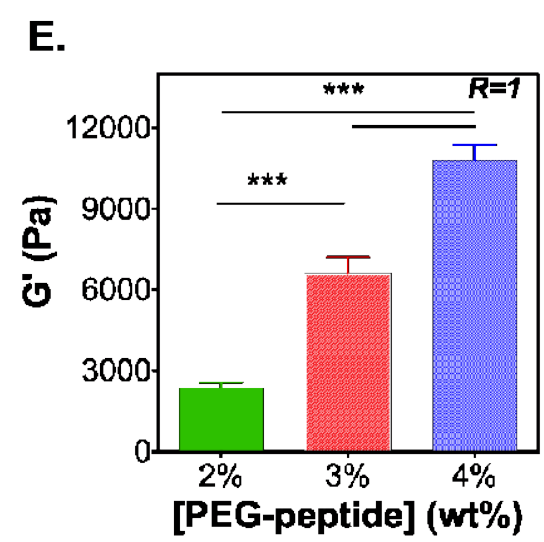

Figure 2. (A) Schematic of SrtA $\mathrm{A}_{7 \mathrm{M}}$-mediated hydrogel crosslinking. (B) In situ rheometry of PEGpeptide (2 wt\%, RGGGG:LPRTG $=1$ ) hydrogel crosslinking. (C) Influence of SrtA ${ }_{7 M}$ concentration on the modulus of PEG-peptide hydrogels ( $3 \mathrm{wt} \%, \mathrm{R}_{\mathrm{GGGG}: \mathrm{LPRTG}}=1$ ) after 10 minutes of gelation. (D) Influence of gelation time on the modulus of PEG-peptide hydrogels ( $\left.3 \mathrm{wt} \%, \mathrm{R}_{\mathrm{GGGG}: \mathrm{LPRTG}}=1\right)$ with $300 \mu \mathrm{M} \mathrm{SrtA} \mathrm{A}_{7 \mathrm{M}}$. (E) Influence of PEG-peptide concentration on the modulus with 10 minutes of gelation time and $300 \mu \mathrm{M} \mathrm{SrtA}_{7 \mathrm{M}}$. 
A.

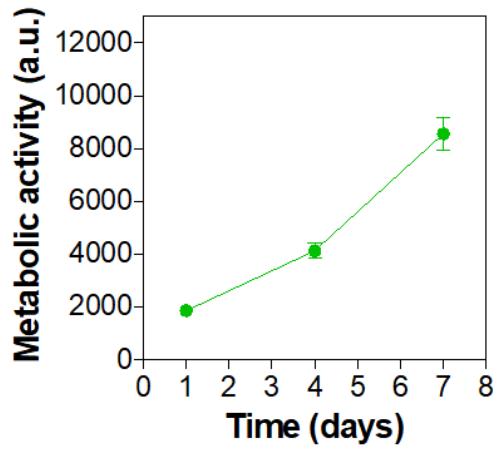

B.
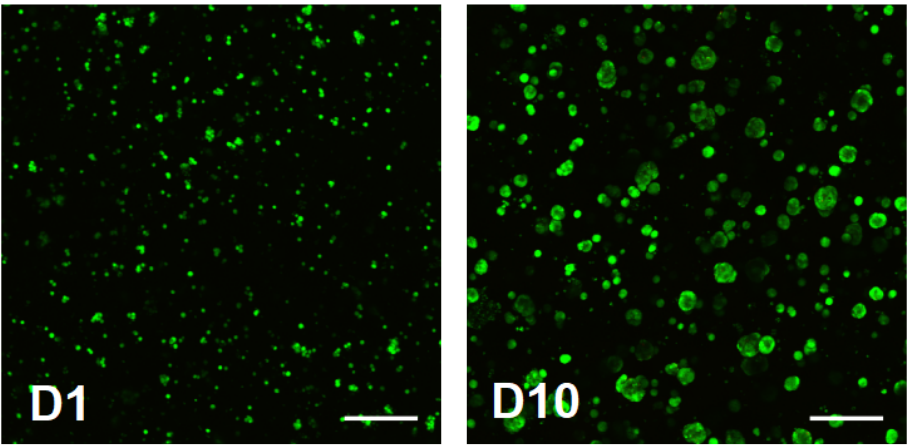

Live/Dead (Scale: $200 \mu \mathrm{m})$

Figure 3. (A) Effect of SrtA $\mathrm{AM}_{7 \mathrm{M}}$-mediated crosslinking on the metabolic activity of encapsulated MIN6 cells $(n=3)$. (B) Representative confocal z-stack images of live/dead stained MIN6 cells encapsulated in PEG-peptide hydrogels. Cells were encapsulated at $2 \times 10^{6}$ cells $/ \mathrm{ml}$ in PEGpeptide hydrogels ( $3 \mathrm{wt} \%, \mathrm{R}_{\mathrm{GGGG}: \mathrm{LPRTG}}=1$ ) and imaged on D1 and D10 post-encapsulation. At least three $z$-stacked images per gel (100 $\mu \mathrm{m}$ thick, 10 slices) were taken. 
A.
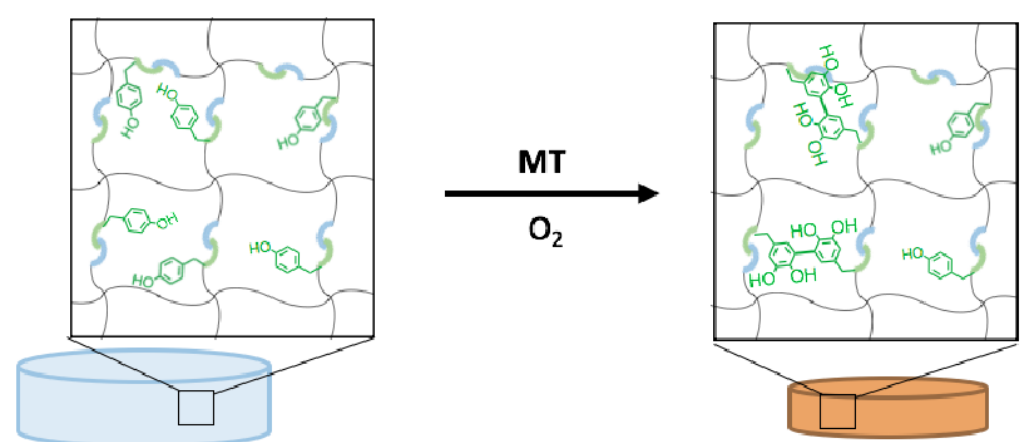

B.

C.
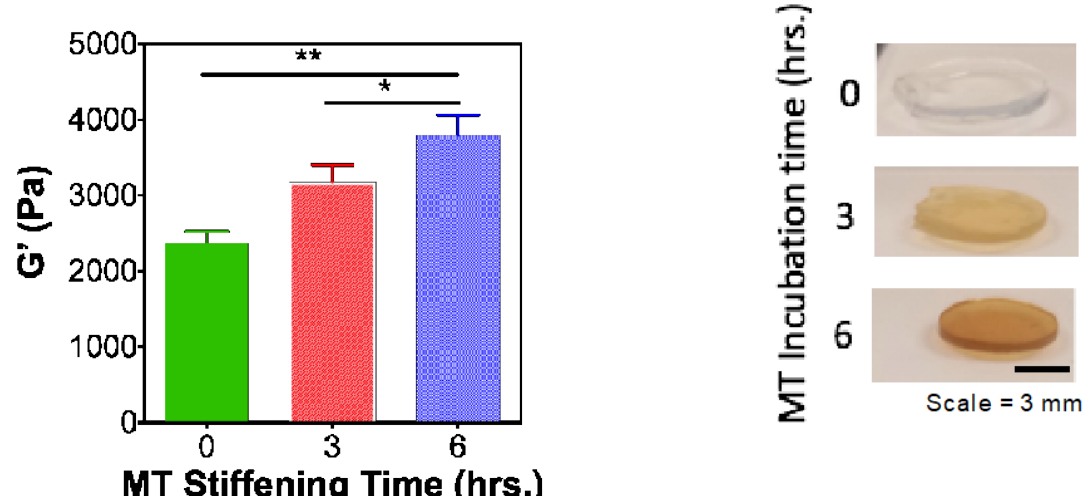

Figure 4. (A) Schematic of MT-triggered secondary crosslinking. (B) Influence of MT incubation time on the modulus of PEG-peptide hydrogels (2 wt\%, $\mathrm{R}_{\mathrm{GGGG} \text { :LPRTG }}=1, \mathrm{MT}=1 \mathrm{kU} / \mathrm{mL}$ ). (C) Representative images of hydrogels incubated with MT for varying duration. 
A.

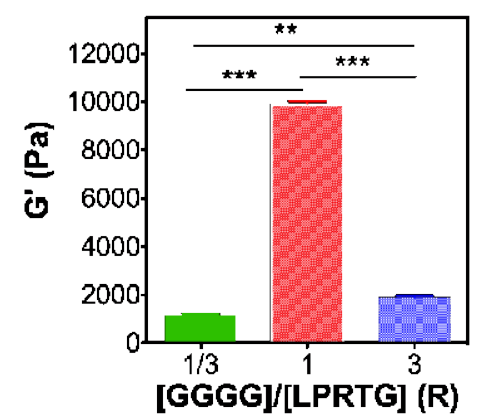

B.

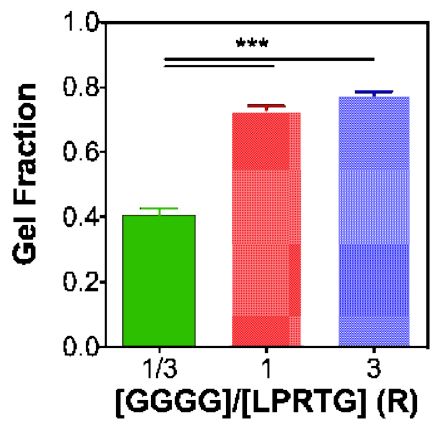

C.

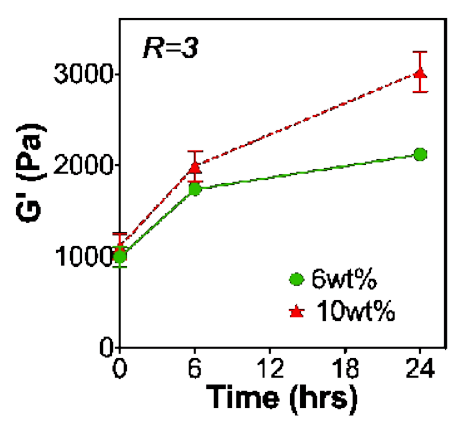

Figure 5. (A) Influence of $R_{G G G G: L P R T G}$ on hydrogel modulus. The gap sizes utilized for $R_{G G G G: L P R T G}$ $=1 / 3, R_{G G G G: L P R T G}=1, R_{G G G G: L P R T G}=3$ were $475 \mu \mathrm{m}, 525 \mu \mathrm{m}$, and $700 \mu \mathrm{m}$, respectively. (B) Influence of R $_{\text {GGGG:LPRTG }}$ on gel fraction. (C) MT-triggered stiffening of PEG-peptide hydrogels $\left(R_{G G G G: L P R T G}=3\right)$. 
A.
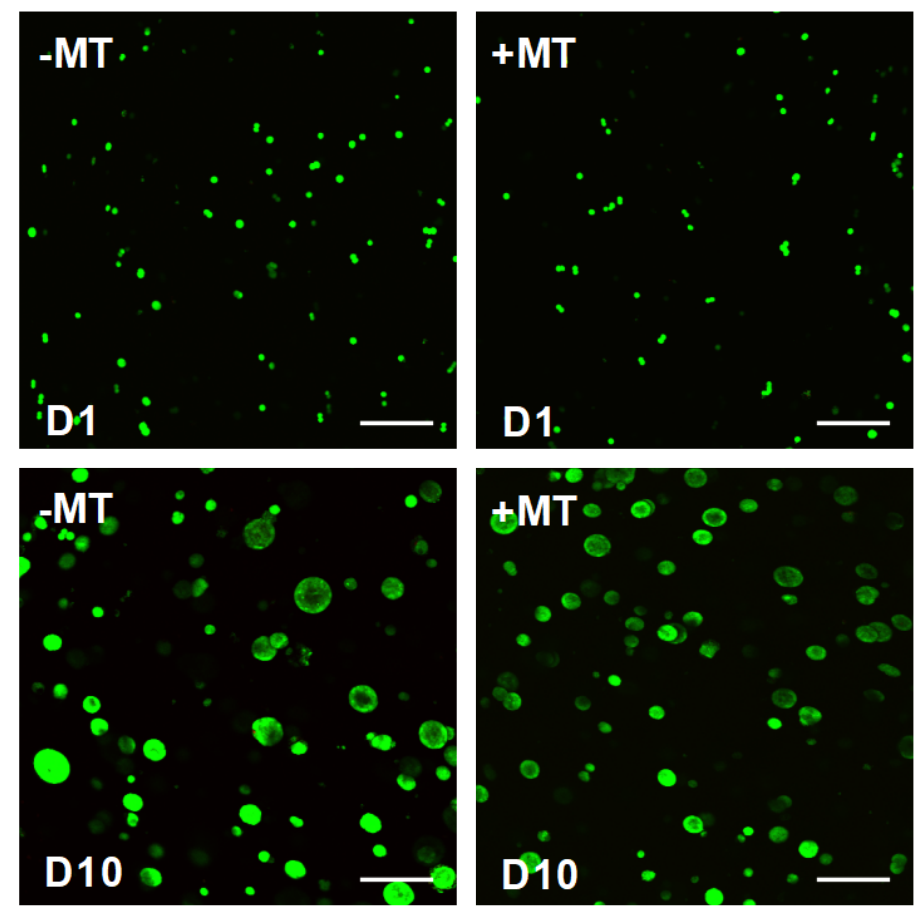

Live/Dead (Scale: $200 \mu \mathrm{m}$ )

B.

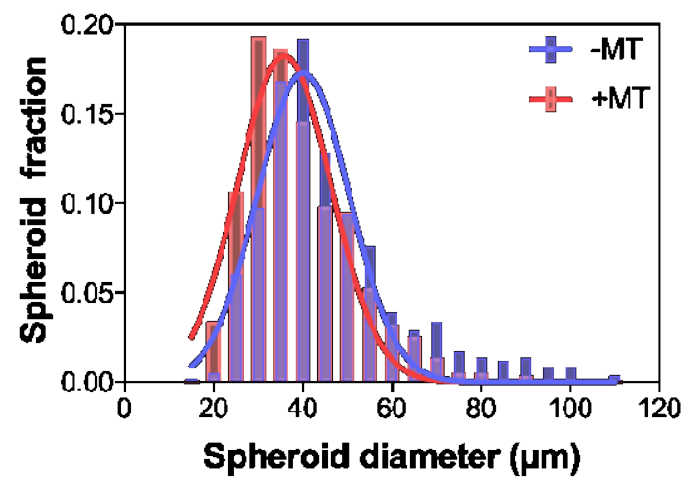

Figure 6. (A) Day 1 and Day 10 live/dead images of encapsulated COLO-357 cells (2x10 cells $/ \mathrm{mL})$ with and without 6 hour incubation with MT $(1 \mathrm{kU} / \mathrm{mL})$. At least three z-stacked images per gel (100 $\mu$ m thick, 10 slices) were taken. (B) Distribution of cell spheroids diameters with or without MT-triggered stiffening. Spheroid sizes were quantified using live/dead staining images at 10-days post-encapsulation. The diameter of spheroids with and without MT-triggered stiffening were $45.91 \pm 0.67 \mu \mathrm{m}(\mathrm{n}=594)$ and $39.48 \pm 0.51 \mu \mathrm{m}(\mathrm{n}=590)$, respectively. A two-tailed t-test was performed for statistical analysis $(p<0.01)$. 


\section{Table of content graphic}

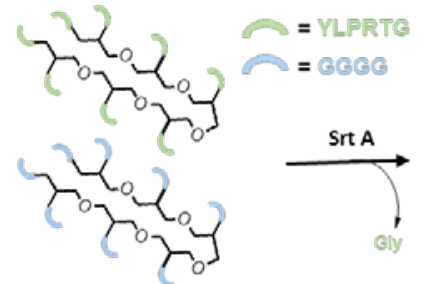

8-Arm PEG-peptide

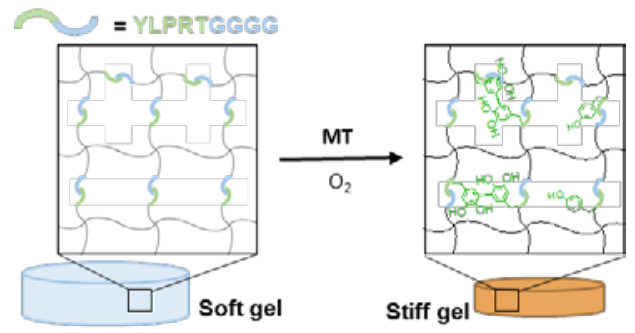

A biocompatible PEG-peptide hydrogel with dynamically tunable stiffness was developed through sortase A-mediated crosslinking and mushroom tyrosinase-triggered stiffening. 


\section{References}

1. K. M. Mabry, R. L. Lawrence and K. S. Anseth, Biomaterials, 2015, 49, 47-56.

2. S. R. Caliari, M. Perepelyuk, B. D. Cosgrove, S. J. Tsai, G. Y. Lee, R. L. Mauck, R. G. Wells and J. A. Burdick, Sci Rep, 2016, 6, 21387.

3. S. Khetan, M. Guvendiren, W. R. Legant, D. M. Cohen, C. S. Chen and J. A. Burdick, Nat Mater, 2013, 12, 458-465.

4. A. M. Rosales, K. M. Mabry, E. M. Nehls and K. S. Anseth, Biomacromolecules, 2015, 16, 798-806.

5. P. C. Georges, J. J. Hui, Z. Gombos, M. E. McCormick, A. Y. Wang, M. Uemura, R. Mick, P. A. Janmey, E. E. Furth and R. G. Wells, Am J Physiol Gastrointest Liver Physiol, 2007, 293, G11471154.

6. K. R. Levental, H. Yu, L. Kass, J. N. Lakins, M. Egeblad, J. T. Erler, S. F. Fong, K. Csiszar, A. Giaccia, W. Weninger, M. Yamauchi, D. L. Gasser and V. M. Weaver, Cell, 2009, 139, 891-906.

7. M. Guvendiren and J. A. Burdick, Nat Commun, 2012, 3, 792.

8. R. S. Stowers, S. C. Allen and L. J. Suggs, Proc Natl Acad Sci U S A, 2015, 112, 1953-1958.

9. Á. N. B. Gyarmati, A. Szilágyi, European Polymer Journal, 2013, 49, 18.

10. H. Shih, Lin C.C, Journal of Materials Chemistry B, 2016, 4, 6.

11. J. J. Roberts, P. Naudiyal, K. S. Lim, L. A. Poole-Warren and P. J. Martens, Biomater Res, 2016, 20, 30.

12. D. Huber, G. Tegl, M. Baumann, E. Sommer, E. G. Gorji, N. Borth, G. Schleining, G. S. Nyanhongo and G. M. Guebitz, Carbohydr Polym, 2017, 157, 814-822.

13. F. Anjum, P. S. Lienemann, S. Metzger, J. Biernaskie, M. S. Kallos and M. Ehrbar, Biomaterials, 2016, 87, 104-117.

14. E. Cambria, K. Renggli, C. C. Ahrens, C. D. Cook, C. Kroll, A. T. Krueger, B. Imperiali and L. G. Griffith, Biomacromolecules, 2015, 16, 2316-2326.

15. N. Z. Alarake, P. Frohberg, T. Groth and M. Pietzsch, Int J Artif Organs, 2017, 40, 159-168.

16. A. Ranga, M. P. Lutolf, J. Hilborn and D. A. Ossipov, Biomacromolecules, 2016, 17, 1553-1560.

17. G. Rocasalbas, A. Francesko, S. Tourino, X. Fernandez-Francos, G. M. Guebitz and T. Tzanov, Carbohydr Polym, 2013, 92, 989-996.

18. H. Y. Liu, T. Greene, T. Y. Lin, C. S. Dawes, M. Korc and C. C. Lin, Acta Biomater, 2017, 48, 258269.

19. E. J. Land, C. A. Ramsden and P. A. Riley, Acc Chem Res, 2003, 36, 300-308.

20. M. W. Popp and H. L. Ploegh, Angew Chem Int Ed Engl, 2011, 50, 5024-5032.

21. Z. Wu, X. Guo and Z. Guo, Chem Commun (Camb), 2011, 47, 9218-9220.

22. L. K. Swee, C. P. Guimaraes, S. Sehrawat, E. Spooner, M. I. Barrasa and H. L. Ploegh, Proc NatI Acad Sci U S A, 2013, 110, 1428-1433.

23. B. M. Paterson, K. Alt, C. M. Jeffery, R. I. Price, S. Jagdale, S. Rigby, C. C. Williams, K. Peter, C. E. Hagemeyer and P. S. Donnelly, Angew Chem Int Ed Engl, 2014, 53, 6115-6119.

24. K. W. Clancy, J. A. Melvin and D. G. McCafferty, Biopolymers, 2010, 94, 385-396.

25. J. Valdez, C. D. Cook, C. C. Ahrens, A. J. Wang, A. Brown, M. Kumar, L. Stockdale, D. Rothenberg, K. Renggli, E. Gordon, D. Lauffenburger, F. White and L. Griffith, Biomaterials, 2017, 130, 90-103.

26. C. C. Lin, C. S. Ki and H. Shih, J Appl Polym Sci, 2015, 132.

27. M. D. Witte, T. Wu, C. P. Guimaraes, C. S. Theile, A. E. Blom, J. R. Ingram, Z. Li, L. Kundrat, S. D. Goldberg and H. L. Ploegh, Nat Protoc, 2015, 10, 508-516.

28. B. D. Fairbanks, M. P. Schwartz, A. E. Halevi, C. R. Nuttelman, C. N. Bowman and K. S. Anseth, Adv Mater, 2009, 21, 5005-5010.

29. B. D. Fairbanks, M. P. Schwartz, C. N. Bowman and K. S. Anseth, Biomaterials, 2009, 30, 67026707. 
30. C. P. Guimaraes, M. D. Witte, C. S. Theile, G. Bozkurt, L. Kundrat, A. E. Blom and H. L. Ploegh, Nat Protoc, 2013, 8, 1787-1799.

31. H. Hirakawa, S. Ishikawa and T. Nagamune, Biotechnol J, 2015, 10, 1487-1492.

32. Q. Wu, H. L. Ploegh and M. C. Truttmann, ACS Chem Biol, 2017, 12, 664-673.

33. C. C. Lin, A. Raza and H. Shih, Biomaterials, 2011, 32, 9685-9695.

34. C. C. Lin and K. S. Anseth, Proc Natl Acad Sci U S A, 2011, 108, 6380-6385.

35. A. Raza and C. C. Lin, J Vis Exp, 2012, DOI: 10.3791/50081, e50081.

36. M. W. Tibbitt and K. S. Anseth, Biotechnol Bioeng, 2009, 103, 655-663.

37. C. C. Lin and K. S. Anseth, Pharm Res, 2009, 26, 631-643.

38. I. M. Chung, N. O. Enemchukwu, S. D. Khaja, N. Murthy, A. Mantalaris and A. J. Garcia, Biomaterials, 2008, 29, 2637-2645.

39. M. P. Lutolf, J. L. Lauer-Fields, H. G. Schmoekel, A. T. Metters, F. E. Weber, G. B. Fields and J. A. Hubbell, Proc Natl Acad Sci U S A, 2003, 100, 5413-5418.

40. Y. Hao, H. Shih, Z. Munoz, A. Kemp and C. C. Lin, Acta Biomater, 2014, 10, 104-114.

41. K. Strijbis, E. Spooner and H. L. Ploegh, Traffic, 2012, 13, 780-789.

42. I. Chen, B. M. Dorr and D. R. Liu, Proc Natl Acad Sci U S A, 2011, 108, 11399-11404.

43. X. Huang, A. Aulabaugh, W. Ding, B. Kapoor, L. Alksne, K. Tabei and G. Ellestad, Biochemistry, 2003, 42, 11307-11315.

44. D. J. Williamson, M. E. Webb and W. B. Turnbull, Nat Protoc, 2014, 9, 253-262.

45. Y. M. Li, Y. T. Li, M. Pan, X. Q. Kong, Y. C. Huang, Z. Y. Hong and L. Liu, Angew Chem Int Ed Engl, 2014, 53, 2198-2202.

46. F. Liu, E. Y. Luo, D. B. Flora and A. R. Mezo, J Org Chem, 2014, 79, 487-492.

47. H. Shih, T. Greene, M. Korc and C. C. Lin, Biomacromolecules, 2016, 17, 3872-3882. 